\title{
A Mini Review of Plant-Based Diets in Hemodialysis
}

\author{
Léonie Dupuis $^{\mathrm{a}}$ Amanda Brown-Tortorici ${ }^{\mathrm{b}}$ Kamyar Kalantar-Zadeh ${ }^{\mathrm{c}}$ \\ Shivam Joshid, e
}

aUniversity of Central Florida College of Medicine, Orlando, FL, USA; ${ }^{\text {bS }}$ usan Samueli Center Integrative Health Institute, University of California Irvine, Costa Mesa, CA, USA; 'Division of Nephrology and Hypertension and Kidney Transplantation, School of Medicine, University of California, Irvine, Orange, CA, USA; dDepartment of Medicine, New York University Grossman School of Medicine, New York, NY, USA; ${ }^{\text {Department }}$ of Medicine, NYC Health and Hospitals/Bellevue, New York, NY, USA

\section{Key Messages}

- Plant-based diets decrease inflammatory markers and have been associated with a reduction in allcause mortality in patients on hemodialysis in observational studies.

- High fiber intake significantly reduces uremic toxins and increases fecal bulk, facilitating the excretion of potassium, which may be one of several mechanisms associated with plant-based foods in tempering the rise in serum potassium.

- Plant protein is of adequate quantity and quality in a diverse, well-planned plant-based diet.

- Lower intake of plant-based food, including less fruits and vegetables, in dialysis patients is associated with constipation, with one study identifying $71.7 \%$ of patients on dialysis with constipation. Constipation may be related to higher likelihood of hyperkalemia.

\section{Keywords}

Plant-based diets · Hemodialysis · Chronic kidney disease · Nutrition

\begin{abstract}
Background: Plant-based diets are defined as an eating pattern focused on the consumption of unprocessed fruits, vegetables, nuts, whole grains, oils, and beans. They can include various forms of vegetarian, vegan, DASH, and Mediterranean diets. Plant-based diets have proven useful in the prevention and treatment of several lifestyle diseases like type 2 diabetes, hypertension, and obesity. Recently, a growing body of literature has emerged regarding plant-based diets
\end{abstract}

karger@karger.com

(c) 2021 S. Karger AG, Basel

www.karger.com/bpu

Karger for patients with kidney failure, including those on dialysis. Although evidence is still limited, preliminary findings are encouraging. Summary: This article reviews current literature on the use of plant-based diets in the treatment of patients on dialysis.

(c) 2021 S. Karger AG, Basel

\section{Introduction to Plant-Based Diets in Hemodialysis}

A plant-based diet is defined as the consumption of plants such as fruits, vegetables, nuts, seeds, oils, whole grains, legumes, and beans. It may or may not include small amounts of animal products such as meat, fish, sea- 
food, eggs, and dairy, but these foods tend to be avoided. Plant foods are rich in phytochemicals, antioxidants, vitamins, and minerals which prove beneficial to health on a micronutrition level, especially when plants are consumed in their whole, unprocessed form. When brought to the forefront of a patient's dietary pattern, these naturally nutritious foods may translate into clinically significant improvements to patients' quality of life and disease prognosis, and mitigate disease complications. Historically, these foods have been avoided in patients on dialysis due to concerns of hyperkalemia and protein deficiency. However, the recent recognition of their potential benefits and improved understanding of potential pitfalls have favored the reintroduction of these foods into the diet of patients on dialysis.

\section{Potential Benefits of Plant-Based Diets in Hemodialysis}

Traditional dietary interventions in patients with kidney disease encourage the restriction of phosphorus, potassium, sodium, and fluid as well as tailoring protein intake to the patient's CKD stage and whether they require kidney replacement therapy. These guidelines tend to result in a diet devoid of fruits and vegetables, lacking in variety and resulting in limited satisfaction [1]. Broadening the diet of patients with kidney disease to include these otherwise healthy foods may not only improve satisfaction and increase variety but may also carry additional benefits.

A recent multinational cohort study $(n=8,078)$ found that higher consumption of fruits and vegetables in patients on dialysis was associated with lower all-cause and noncardiovascular death [2]. In this study, patients receiving long-term hemodialysis completed a food frequency questionnaire for a median follow-up of 2.7 years (18,586 person-years). Only $4 \%$ of the patients consumed at least 4 servings of fruit and vegetables per day as recommended in the general population.

Plant-based diets, a fiber- and polyphenol-rich intervention, may attenuate cardiovascular disease risk markers in patients on hemodialysis. Polyphenols are a diverse group of micronutrients only consumed through plants. A systematic review and meta-analysis of 12 studies found that polyphenol-rich interventions in patients on hemodialysis improved diastolic blood pressure $(p=0.0001)$, triglycerides $(p=0.01)$, and myeloperoxidase $(p=0.0001)$ [3]. Fiber supplementation (results described in more detail in the "Fiber and Uremic Toxins" section) was found to significantly lower total cholesterol, LDL cholesterol, and inflammatory markers - all of which are known cardiovascular risk factors [4].

Plant fats, which are rich in unsaturated fatty acids, have shown to be beneficial in the diets of patients on hemodialysis by improving inflammatory markers. Vegetable oils are rich in polyunsaturated fatty acids, such as linoleic acid, which are known to have cardioprotective and anti-inflammatory properties. In one study, linoleic acid levels, as a proportion of plasma fatty acids, were shown to be inversely associated with IL- 6 and all-cause mortality in a cross-sectional study of hemodialysis patients [5].

\section{Benefits of Plant-Based Diets in the Complications of Hemodialysis}

\section{Fiber and Uremic Toxins}

Dietary fiber is a nondigestible, nonabsorbable carbohydrate polymer that may be used to treat chronic kidney disease by exploiting its ability to reduce uremic toxin production and, perhaps, postpone dialysis [6]. Since dietary fiber is exclusively found in plants, there may be a unique advantage associated with the use of plant-based diets (Table 1).

Dietary fiber is the foundational element to gut microbial growth. Fermentable fibers are metabolized into short-chain fatty acids, especially acetate, propionate, and butyrate [7]. Short-chain fatty acids may play a role in kidney health by modulating systemic inflammation (and anti-inflammatory responses) and maintaining the integrity of the gut mucosal barrier [7]. Today, plant-derived complex carbohydrates are recognized as prebiotics for their role in promoting the growth and metabolic activity of beneficial saccharolytic organisms like Bifidobacteria and Lactobacillus [8]. The protective effects of gut microbiota have been widely studied in chronic kidney disease, but there is a paucity in data regarding the bidirectional gut-kidney axis in hemodialysis patients. Compared to healthy individuals, a cross-sectional study $(n=24)$ found end-stage kidney disease patients on hemodialysis to have an increased $(p<0.02)$ relative abundance of Proteobacteria (mainly Gammaproteobacteria), Actinobacteria, and Firmicutes by phylogenic microarrays [9]. Of note, these taxa are not usually associated with uremic toxin production.

Indoxyl sulfate and p-cresol are uremic toxins generated by colonic bacteria in response to food that is not absorbed in the small bowel. Uremic toxins accumulate 
Table 1. Potential benefits of plant-based foods in patients on dialysis

The consumption of plant-based diets (or fiber) in patients on hemodialysis has been associated with the following:
Lower uremic toxin production

Decreased all-cause mortality and noncardiovascular mortality

Reduced inflammatory markers

Favorable changes in the gut microbiome

A lower frequency of major adverse cardiovascular events

Reduced constipation

Increased fecal bulk in patients with chronic kidney disease and may contribute to the progressive loss of glomerular filtration rate by increasing the expression of transforming growth factor beta which increases fibrogenesis [10]. A cross-sectional study of patients undergoing hemodiafiltration found that vegetarian patients had $47 \%$ lower levels of indoxyl sulfate and $67 \%$ lower levels of p-cresyl sulfate than the nonvegetarian patients [11]. In another study of patients on hemodialysis, an increase in fiber intake was shown to significantly reduce plasma levels of indoxyl sulfate by $17 \%(p=0.04)$ and nonsignificantly reduce plasma levels of p-cresol by $8 \%(p=0.63)$ in a randomized control trial $(n=56)[12]$. Another cross-sectional study $(n=175)$ found that increased levels of $\mathrm{p}$-cresol were associated with an increased risk of death in patients on maintenance hemodialysis [13].

Outside of decreasing uremic toxins, fiber supplementation in patients on hemodialysis also improved lipid profiles and oxidative status and decreased systemic inflammation when compared to a placebo in a 6-week randomized control trial $(n=124)$ [4]. The results of the trial revealed a significant $(p<0.05)$ decrease in total cholesterol (TC) level, high-density lipoprotein (HDL) level, and TC:LCL ratio; a significant $(p<0.05)$ decrease in total antioxidant capacity and malondialdehyde; and a significant $(p<0.05)$ decrease in tumor necrosis factor- $\alpha$, interleukin-6, interleukin-8, and C-reactive protein in the 10 and $20 \mathrm{~g}$ per day fiber supplement groups [4]. A longterm prospective cohort study of patients on peritoneal dialysis $(n=881)$ showed an independent association between fiber intake and all-cause mortality wherein each gram per day increase in fiber intake correlated with a $13 \%$ reduction in all-cause mortality [14].

Contrarily, low dietary fiber intake has been shown to negatively impact patients on hemodialysis on multiple occasions. In a prospective cohort study of patients on dialysis $(n=127)$, lower fiber intake was associated with an increased risk for major adverse cardiovascular events (adjusted hazard ratio: 1.78; 95\% CI: 1.13-2.80) when compared to patients with higher fiber intake [15]. Another study on maintenance hemodialysis patients $(n=$ 128) found that dietary fiber level was independently correlated with advanced glycation end products $\left(r^{2}=0.164\right.$, $p=0.017)$ and C-reactive protein levels $\left(r^{2}=0.238, p=\right.$ $0.01)[16]$.

\section{Constipation}

In hemodialysis patients, the incidence of constipation varied according to the definition of constipation, but it was noted to be $71.7 \%$ in a 2013 cross-sectional study using the Roma III criteria $(n=478)$ [17]. As a chronic or frequently recurrent symptom, constipation impairs patients' health-related quality of life and may result in a substantial emotional and social burden [18]. The issue may be easily treated by emphasizing the consumption of high-fiber foods, like plants. It may be possible that the historically low consumption of fiber-rich foods of patients on hemodialysis may be to blame for the high rate of constipation in this population.

In the general population, patients with constipation had $12 \%$ higher all-cause mortality, $11 \%$ higher incidence of coronary heart disease, and 19\% higher incidence of ischemic stroke compared to patients without constipation [19]. US Veterans using one or $>2$ types of laxatives experienced a similarly higher risk of all-cause mortality and cardiovascular and cerebrovascular burden when compared to US veterans not taking any laxatives. A recent multicenter study $(n=305)$ evaluated the prevalence and correlates of constipation in hemodialysis patients from 4 dialysis centers using a questionnaire based on the Roma IV criteria to assess constipation status and lifestyle habits along with a food frequency ques- 
tionnaire focused on the main sources of dietary fiber and found that $>30 \%$ of patients had constipation at a given point in time [20]. In this study, the independent predictors of constipation symptoms included diabetes and lower frequency of fruit intake [20]. Moreover, constipation can worsen hyperkalemia, while having soft bowel movement may be associated with a lower likelihood of hyperkalemia [21].

\section{Hyperphosphatemia}

Although phosphorus is an essential nutrient, it is difficult for patients to avoid states of phosphate excess in advanced CKD. Consequently, patients must be placed on phosphorus-restricted diets to reduce the risk of hyperphosphatemia, which has been associated with an increased risk of death and cardiovascular disease. Given the varying bioavailability of phosphorus from foods, dietary changes may be made to take advantage of low phosphate-containing foods. In the gut, $40-60 \%$ of phosphate in animal protein is absorbed by the intestinal tract compared to only $10-30 \%$ of phosphorus in plant protein [22]. This is partly because the bulk of phosphorus in plants is bound to phytate, which is difficult for humans to digest due to lack of phytase to release the phosphorus [23].

In a cross-over trial in patients with $\mathrm{CKD}$, the effects of vegetarian and meat diets on phosphorus balance showed that 1 week of a vegetarian diet led to significantly lower serum phosphorus $(p=0.02)$ and significantly lower fibroblast growth factor-23 levels $(p=0.008)$ than being on a meat diet [24]. This finding has also been seen in a small observational study by $\mathrm{Wu}$ et al. [25] wherein serum phosphate levels were significantly $(p<0.05)$ lower in vegetarian patients $(4.1 \mathrm{mg} / \mathrm{dL} \pm 0.2)$ than in nonvegetarian patients $(4.8 \mathrm{mg} / \mathrm{dL} \pm 0.1)$ on hemodialysis.

\section{Vitamin K and Vitamin B12}

When diagnosed with end-stage kidney disease, traditional dietary interventions may place patients at risk for vitamin deficiencies. These deficiencies arise due to a wide range of causes including biological factors such as decreased appetite, decreased smell, and decreased taste and behavioral factors such as difficulty cooking, difficulty obtaining ingredients, and difficulty following dietary regimens.

There is a general recommendation that anyone following a plant-based diet should supplement vitamin B12 in order to prevent deficiency [10]. Safe levels of vitamin B12 in patients on hemodialysis have yet to be determined, but regular monitoring of patients who take vita- min B12 supplements can help identify potential adverse effects.

Particularly, patients on dialysis (both peritoneal dialysis and maintenance hemodialysis) are at risk of vitamin K deficiency, which has been associated with vascular calcification, bleeding risk, and cardiovascular risk in the general population and patients on dialysis $[10,26]$. Vitamin $\mathrm{K}$ is mainly present in green vegetables such as spinach, kale, Brussels sprouts, and broccoli, so dietary regimens that reduce consumption of plant foods may contribute to the development or worsening of vitamin $\mathrm{K}$ deficiency [10]. It is important to know that patients on warfarin should not be deprived of vitamin K-rich vegetables [27]. Expert guidelines recommend a stable intake of vitamin K-rich foods, which is often misinterpreted as "no K-rich food" [27].

\section{Potential Pitfalls of Plant-Based Diets in Kidney Failure}

\section{Hyperkalemia}

One of the biggest concerns when transitioning a patient on hemodialysis to a plant-based diet is the risk for hyperkalemia. It is thus recommended that patients on dialysis should be placed on a potassium-restricted diet to minimize the risk of hyperkalemia, which often results in the omissions of plant-based foods. However, many of the case reports that link hyperkalemic events to foods (and not potassium-based salts or additives) were the result of the consumption of a juice, sauce, or dried fruit [28]. These foods, in particular, are all in formats that concentrate the potassium content of the food when compared to its whole-food form and facilitate a higher rate of potassium ingestion. The whole-food source is less densely composed of potassium and limits total potassium consumption. Further, fiber increases fecal bulk, facilitating excretion of potassium with bowel movements, potentially tempering a rise in serum potassium [29]. Both juices and sauces may be prepared in forms of limited or no fiber, which may facilitate hyperkalemia.

In observational studies, little to no association has been seen between dietary potassium and serum potassium among patients on dialysis [29]. The balancewise cross-sectional study $(n=140)$ compared dietary potassium intake with serum potassium levels and found no association between the 2 [29]. Another study found that reported dietary potassium may only explain $2 \%$ of the variance in mean predialysis potassium levels [30]. 
In regard to the consumption of plant foods by patients on dialysis and hyperkalemia, Saglimbene et al. [2] conducted a prospective observational study $(n=8,078)$ and stratified participants into tertiles based on servings of fruit and vegetable intake per week. The tertiles corresponded to $0-5.5(n=2,520), 5.6-10(n=5.6-10)$, and $>10(n=2,600)$ servings of fruits and vegetables per week [2]. The predialysis potassium levels for each tertile, respectively, were 5.1,5.0, and 5.0, showing very similar potassium levels across a wide range of plant intake [2]. In another study by $\mathrm{Wu}$ et al. [25], potassium levels among vegetarian hemodialysis patients $(5.0 \mathrm{mg} / \mathrm{dL} \pm 0.2)$ and nonvegetarian hemodialysis patients $(4.8 \mathrm{mg} / \mathrm{dL} \pm 0.12)$ were not significantly different. Gonzalez-Ortiz et al. [31] also conducted a prospective study $(n=150)$ on patients undergoing hemodialysis and found that a higher healthy plant-based diet score was not associated with the odds of hyperkalemia (potassium $>5.5 \mathrm{mEq} / \mathrm{L}$; OR 1.00 ; $95 \% \mathrm{CI}$ : 0.94-1.07).

The overall lack of randomized control trials in hemodialysis patients limits conclusive statements regarding potassium restriction in patients on dialysis. As such, hyperkalemia remains a concern in the management of any patient on hemodialysis, regardless of their dietary choices. Nonetheless, studies on patients undergoing hemodialysis while eating plant-based diets have not shown increased risks of hyperkalemia [2, 25, 29, 31]. More prospective studies need to be conducted to draw more definitive conclusions on hyperkalemia and plant-based diets in hemodialysis.

\section{Protein Quality and Quantity}

Another concern with a plant-based diet is the quality and quantity of protein intake. The recently released $\mathrm{Na}$ tional Kidney Foundation's Kidney Disease Outcome Quality Initiative Clinical Practice Guideline for Nutrition in CKD recommends a protein intake $1.0-1.2 \mathrm{~g} / \mathrm{kg}$ of body weight per day in patients on maintenance dialysis who are metabolically stable [32]. Patients on dialysis meet or exceed these requirements when consuming a plant-based diet without signs of undernutrition. In 2 studies, patients on dialysis consuming a vegetarian diet were shown to consume $1.2-1.25 \mathrm{~g} / \mathrm{kg} /$ day $[11,25]$. Plant and animal protein sources are composed of different proportions of amino acids and have varying availability to the human body [33]. These differences, although biochemically relevant, have been shown to be trivial in clinical context so long as there is variety and sufficiency of foods in the plant-based diet [34]. The idea that plant proteins need to be paired complementarily based on their amino acid profiles to prevent amino acid deficiencies is outdated as the body stores amino acids for hours to days at a time $[34,35]$. By eating a wide variety of plants, patients on dialysis can avoid issues related to protein quantity and quality.

\section{Conclusion}

Plant-based diets may benefit patients on hemodialysis by mitigating the complications of kidney failure while providing a varied and nutritious alimentation. Plantbased diets may reduce the risk for metabolic acidosis or hyperphosphatemia, reduce levels of uremic toxins, lower the likelihood of constipation, and provide sufficient vitamin $\mathrm{K}$. There is a paucity of studies exploring plantbased diets in patients on hemodialysis and more randomized control trials need to be conducted to investigate the benefits and risks of plant-based diets in this patient population.

\section{Acknowledgement}

All the authors contributed to this article.

\section{Statement of Ethics}

This study is exempt from Ethical Committee approval as it is a review of existing literature.

\section{Conflict of Interest Statement}

The authors have no conflicts of interest to declare.

\section{Funding Sources}

No financial support was procured for the creation of this study.

\section{Author Contributions}

Leonie Dupuis is the primary author and drafted the manuscript. Amanda Brown-Tortorici and Kamyar Kalantar-Zadeh contributed to the section "Benefits of Plant-based Diets in the Complications of Kidney Disease." Kamyar Kalantar-Zadeh and Shivam Joshi provided revisions to the scientific content of the manuscript. 


\section{References}

1 Kalantar-Zadeh K, Tortorici AR, Chen JL, Kamgar M, Lau WL, Moradi H, et al. Dietary restrictions in dialysis patients: is there anything left to eat? Semin Dial. 2015 Mar-Apr; 28(2):159-68

2 Saglimbene VM, Wong G, Ruospo M, Palmer SC, Garcia-Larsen V, Natale P, et al. Fruit and vegetable intake and mortality in adults undergoing maintenance hemodialysis. Clin J Am Soc Nephrol. 2019 Feb 7;14(2):250-60.

3 Marx W, Kelly J, Marshall S, Nakos S, Campbell $\mathrm{K}$, Itsiopoulos C. The effect of polyphenol-rich interventions on cardiovascular risk factors in haemodialysis: a systematic review and metaanalysis. Nutrients. 2017 Dec 11;9(12):1345.

4 Xie LM, Ge YY, Huang X, Zhang YQ, Li JX. Effects of fermentable dietary fiber supplementation on oxidative and inflammatory status in hemodialysis patients. Int J Clin Exp Med. 2015;8(1):1363-9.

5 Huang X, Stenvinkel P, Qureshi AR, Risérus $\mathrm{U}$, Cederholm T, Bárány $\mathrm{P}$, et al. Essential polyunsaturated fatty acids, inflammation and mortality in dialysis patients. Nephrol Dial Transplant. 2012 Sep;27(9):3615-20.

6 Rampton DS, Cohen SL, Crammond VD, Gibbons J, Lilburn MF, Rabet JY, et al. Treatment of chronic renal failure with dietary fiber. Clin Nephrol. 1984 Mar;21(3):159-63.

7 Huang W, Zhou L, Guo H, Xu Y, Xu Y. The role of short-chain fatty acids in kidney injury induced by gut-derived inflammatory response. Metabolism. 2017 Mar;68:20-30.

8 Camerotto C, Cupisti A, D'Alessandro C, Muzio F, Gallieni M. Dietary fiber and gut microbiota in renal diets. Nutrients. 2019 Sep 9; 11(9):2149.

9 Vaziri ND, Wong J, Pahl M, Piceno YM, Yuan J, DeSantis TZ, et al. Chronic kidney disease alters intestinal microbial flora. Kidney Int. 2013 Feb;83(2):308-15

10 Carrero JJ, González-Ortiz A, Avesani CM, Bakker SJL, Bellizzi V, Chauveau P, et al. Plant-based diets to manage the risks and complications of chronic kidney disease. Nat Rev Nephrol. 2020 Sep 1;16(9):525-42.

11 Kandouz S, Mohamed AS, Zheng Y, Sandeman S, Davenport A. Reduced protein bound uraemic toxins in vegetarian kidney failure patients treated by haemodiafiltration. Hemodial Int. 2016 Oct;20(4):610-7.

12 Sirich TL, Plummer NS, Gardner CD, Hostetter TH, Meyer TW. Effect of increasing dietary fiber on plasma levels of colon-derived solutes in hemodialysis patients. Clin J Am Soc Nephrol. 2014 Sep 5;9(9):1603-10.
13 Bammens B, Evenepoel P, Keuleers H, Verbeke K, Vanrenterghem Y. Free serum concentrations of the protein-bound retention solute $\mathrm{p}$ cresol predict mortality in hemodialysis patients. Kidney Int. 2006 Mar;69(6):1081-7.

14 Xu X, Li Z, Chen Y, Liu X, Dong J. Dietary fibre and mortality risk in patients on peritoneal dialysis. Br J Nutr. 2019 Nov 14;122(9): 996-1005.

15 Wang AY, Sea MM, Ng K, Wang M, Chan IH, Lam CW, et al. Dietary fiber intake, myocardial injury, and major adverse cardiovascular events among end-stage kidney disease patients: a Prospective Cohort Study. Kidney Int Rep. 2019 Jun;4(6):814-23.

16 Demirci BG, Tutal E, Eminsoy IO, Kulah E, Sezer S. Dietary fiber intake: its relation with glycation end products and arterial stiffness in end-stage renal disease patients. J Ren Nutr. 2019 Mar;29(2):136-42.

17 Zhang J, Huang C, Li Y, Chen J, Shen F, Yao $\mathrm{Q}$, et al. Health-related quality of life in dialysis patients with constipation: a cross-sectional study. Patient Prefer Adherence. 2013;7: 589-94.

18 Bharucha AE, Pemberton JH, Locke GR 3rd. American gastroenterological association technical review on constipation. Gastroenterology. 2013 Jan;144(1):218-38.

19 Sumida K, Molnar MZ, Potukuchi PK, Thomas F, Lu JL, Yamagata K, et al. Constipation and risk of death and cardiovascular events. Atherosclerosis. 2019 Feb;281:114-20.

20 Dos Santos RG, Scatone NK, Malinovski J, Sczip AC, de Oliveira JC, Morais JG, et al. Higher frequency of fruit intake is associated with a lower risk of constipation in hemodialysis patients: a Multicenter Study. J Ren Nutr. 2021;31(1):85-9.

21 Khedr E, Abdelwhab S, El-Sharkawy M, Ali M, El Said K. Prevalence of hyperkalemia among hemodialysis patients in Egypt. Ren Fail. 2009;31(10):891-8.

22 Noori N, Sims JJ, Kopple JD, Shah A, Colman $\mathrm{S}$, Shinaberger CS, et al. Organic and inorganic dietary phosphorus and its management in chronic kidney disease. Iran J Kidney Dis. 2010 Apr;4(2):89-100.

23 Karp HJ, Vaihia KP, Kärkkäinen MU, Niemistö MJ, Lamberg-Allardt CJ. Acute effects of different phosphorus sources on calcium and bone metabolism in young women: a wholefoods approach. Calcif Tissue Int. 2007 Apr; 80(4):251-8.

24 Moe SM, Zidehsarai MP, Chambers MA, Jackman LA, Radcliffe JS, Trevino LL, et al.
Vegetarian compared with meat dietary protein source and phosphorus homeostasis in chronic kidney disease. Clin J Am Soc Nephrol. 2011 Feb;6(2):257-64.

25 Wu TT, Chang CY, Hsu WM, Wang IK, Hsu $\mathrm{CH}$, Cheng $\mathrm{SH}$, et al. Nutritional status of vegetarians on maintenance haemodialysis. Nephrology. 2011 Aug;16(6):582-7.

26 Cozzolino M, Mangano M, Galassi A, Ciceri P, Messa P, Nigwekar S. Vitamin K in chronic kidney disease. Nutrients. 2019 Jan 14; 11(1):168.

27 Leblanc C, Dubé MP, Presse N, Dumas S, Nguyen M, Rouleau-Mailloux É, et al. Avoidance of vitamin K-rich foods is common among warfarin users and translates into lower usual vitamin $\mathrm{K}$ intakes. J Acad Nutr Diet. 2016 Jun;116(6):1000-7.

28 Te Dorsthorst RPM, Hendrikse J, Vervoorn MT, van Weperen VYH, van der Heyden MAG. Review of case reports on hyperkalemia induced by dietary intake: not restricted to chronic kidney disease patients. Eur J Clin Nutr. 2019 Jan;73(1):38-45.

29 St-Jules DE, Goldfarb DS, Sevick MA. Nutrient non-equivalence: does restricting highpotassium plant foods help to prevent hyperkalemia in hemodialysis patients? J Ren Nutr. 2016 Sep;26(5):282-7.

30 Noori N, Kalantar-Zadeh K, Kovesdy CP, Murali SB, Bross R, Nissenson AR, et al. Dietary potassium intake and mortality in longterm hemodialysis patients. Am J Kidney Dis. 2010 Aug;56(2):338-47.

31 González-Ortiz A, Xu H, Ramos-Acevedo S, Avesani CM, Lindholm B, Correa-Rotter R, et al. Nutritional status, hyperkalaemia and attainment of energy/protein intake targets in haemodialysis patients following plant-based diets: a longitudinal cohort study. Nephrol Dial Transplant. 2020.

32 Ikizler TA, Burrowes JD, Byham-Gray LD, Campbell KL, Carrero JJ, Chan W, et al. KDOQI clinical practice guideline for nutrition in CKD: 2020 update. Am J Kidney Dis. 2020;76(3):S1-S107.

33 Dietary reference intakes for energy, carbohydrate, fiber, fat, fatty acids, cholesterol, protein, and amino acids (macronutrients). $\mathrm{Na}$ tional Academy of Sciences; 2005.

34 Young VR, Pellett PL. Plant proteins in relation to human protein and amino acid nutrition. Am J Clin Nutr. 1994 May;59(5 Suppl): 1203s-12S.

35 Munro H. Free amino acid pools and their role in regulation. 1970 . 